\title{
糖尿病与非酒精性脂肪性肝病
}

\author{
王乾, 赵家军* \\ 山东大学附属山东省立医院内分泌科, 济南 250021 \\ * 联系人, E-mail: jjzhao@sdu.edu.cn \\ 收稿日期：2018-02-13; 接受日期：2018-03-10; 网络版发表日期：2018-06-20
}

\begin{abstract}
摘要 非酒精性脂肪性肝病(NAFLD)在糖尿病(DM)患者中越来越普遍. 糖尿病尤其是2型糖尿病(T2DM)的存在 加速了NAFLD的进展. DM患者中存在肝酶无明显升高的NAFLD患者. NAFLD是糖尿病患者大血管和微血管并 发症发生的独立危险因素。噻唑烷二酮、DPP-4抑制剂及GLP-1类似物可以有效缓解糖尿病患者中脂肪肝的进展, 有些药物在长时间使用后, 可以逆转NAFLD.
\end{abstract}

关键词糖尿病, 非酒精性脂肪肝, 糖尿病并发症, 降糖药

糖尿病(diabetes mellitus, DM)是由遗传和环境因 素共同引起的一组以糖代谢紊乱为主要表现的临床综 合征. 胰岛素缺乏和胰岛素作用障碍单独或同时引起 糖类、脂肪、蛋白质、水和电解质等的代谢紊乱，临 床以慢性高血糖为主要特征，其急性并发症有DM酮 症酸中毒、高渗性高血糖状态和乳酸性酸中毒. DM 可并发多种慢性并发症, 导致器官功能障碍和衰竭, 甚至致残或致死. $\mathrm{DM}$ 目前被认为是全球性的流行病. 据估计，全球有 4 亿多人患有 DM，而国际糖尿病联合 会(international diabetes federation, IDF)预计, 未来30 年这一数字将上升至 6.5 亿 $^{[1]}$.

非酒精性脂肪性肝(nonalcoholic fatty liver disease, NAFLD)和DM这两种常见疾病常常并存, 在有症状和 无症状的DM患者中存在相当多的未诊断的NAFLD, 并且具有共同的发病机制，包括胰岛素抵抗、腹型肥 胖、代谢综合征(MetS)及其组分, 以及活动量不足. 然 而, 与视网膜病变、神经病变和肾病不同, NAFLD目 前是 DM尤其是 2 型糖尿病(T2DM)的一种基本未被普
遍认识的并发症. 内分泌科医师和初级保健医师经常 在临床实践中忽视，使得NAFLD导致DM患者严重的 代谢，心血管 $(\mathrm{CV})$ 和肝脏后果. 了解NAFLD与DM之 间复杂的关系是非常重要的, 以便为这些病情复杂的 患者提供更好的临床治疗和护理.

NAFLD是指除外饮酒和其他明确的肝损伤因素 所致的, 以弥漫性肝细胞大泡性脂肪变为主要特征的 临床病理综合征, 包括单纯性脂肪性肝病以及由其演 变的脂肪性肝炎、脂肪性肝纤维化和脂肪性肝硬化. 因此，NAFLD是一个广泛的疾病谱，而并非一种单纯 的代谢性疾病. 尽管可以通过影像学检查或组织学检 查进行诊断, 但NAFLD和非酒精性脂肪性肝炎(nonalcoholic steatohepatitis, NASH)只能通过肝活检来区分. NAFLD如不及时干预，除进展为肝纤维化、肝硬化 外, 肝细胞癌的发生率也会增加.

\section{1 流行病学}

$\mathrm{DM}$ 是一种以胰岛素抵抗和 $\beta$ 细胞损伤导致高血

引用格式: 王乾, 赵家军. 糖尿病与非酒精性脂肪性肝病. 中国科学: 生命科学, 2018, 48: 876-881

Wang Q, Zhao J J. Diabetes mellitus and non-alcoholic fatty liver disease (in Chinese). Sci Sin Vitae, 2018, 48: 876-881, doi: 10.1360/N052018-00042 
糖症作为标志复杂的代谢疾病 ${ }^{[2]}$. 由于平均预期寿命 的增加, 肥胖的发病率增加以及尤其是发展中国家的 生活方式西化 ${ }^{[3]}$, 其发病率正在迅速上升, 而其长期并 发症是其高发病率、死亡率和特殊的医疗成本的主要 原因 ${ }^{[4]}$.

NAFLD是一个新兴的公共卫生问题，其与肥胖， DM，心血管疾病等慢性病之间关系密切，NAFLD患 者死亡率明显高于年龄和性别匹配的一般人群，恶性 肿瘤、心血管疾病和肝脏并发症是最常见的死亡原 因. 近几年来其患病率急剧增加, 是西方国家最常见的 慢性肝病. 目前NAFLD被认为是MetS的在肝脏的表 现. 研究显示, 超过 $76 \%$ 的 T2DM患者患有NAFLD ${ }^{[5]}$. 在一项通过超声确诊NAFLD的研究中，T2DM患者中 NAFLD的患病率也达到了 $69.4 \%{ }^{[6]}$ ，而在另外一项通 过组织学诊断NAFLD的研究中, 发现组织学诊断的 NASH在T2DM患者中的发病率为 $78 \%{ }^{[7]}$. 上述研究说 明DM和NAFLD的发病密切相关.

值得注意的是，在无NAFLD症状的T2DM患者中 有出现严重的晚期纤维化的情况. Leite等人 ${ }^{[7]}$ 针对 92 例同时患有T2DM和NAFLD患者，92例的患者有 $78 \%$ 被诊断为NASH, $50 \%$ 的患者存在有进展性纤维化. 而 Fracanzani等人 ${ }^{[8]}$ 报道458例患者中, T2DM与NASH和 进展的纤维化有独立于其他风险因素的显著性相关关 系. 这两项研究表明, 在DM患者中, 诊断的NAFLD患 者中, NASH占有较大比例. 在另外一项关于NAFLD患 者的调查中, 诊断为 NASH的患者比其他的轻度脂肪 肝的患者显示出与DM及胰岛素抵抗更高的相关性 ${ }^{[9]}$. 有些研究中在出现更严重NAFLD程度的DM患者中, 甚至转氨酶的程度与无NAFLD的DM患者无差异. 因 此, 血清转氨酶水平不是 $\mathrm{T} 2 \mathrm{DM}$ 患者篮查和诊断 NAFLD的可靠指标，不应该在临床实践中应用 ${ }^{[10]}$. 更 严重的NAFLD程度的相关性提示, DM可能在NAFLD 的疾病过程中起到了推动作用. 另外一项前瞻性调查 为DM在NAFLD疾病的进展中的作用提供了更可靠的 依据 ${ }^{[11]}$ 。该研究纳入了 173643 名DM患者以及 650620 名非DM人群的研究中, 在纳入时, 排除了肝脏疾病. 在至少 10 的随访期之后，NAFLD的发生率在DM患者 中是非DM人的接近两倍(18.13/10000 vs. 9.55/10000). 肝癌的发生率也得到了相似的结果(2.39/10000 vs. $0.87 / 10000)$. DM成为非酒精性脂肪肝以及肝癌发生 的独立危险因素。鉴于NAFLD在DM患者中的高患病
率，以及DM患者中NAFLD的严重性，以及无临床症 状或一般肝功指标异常的NAFLD的出现, 在DM患者 中, NAFLD的诊断和治疗应被视为内分泌专家关注的 重点.

但是 1 型糖尿病 (T1DM) 与NAFLD相关的研究数 据却非常有限. 在一项对 100 名成年T1DM患者的而研 究中, 脂肪肝的检出率为 $11 \%^{[12]}$. 另一项对 692 名埃及 平均时间为 9.6 年的 T1DM儿童的研究中, 超声诊断中 有 $4.5 \%$ 有异常超度和/或肝肿大 ${ }^{[13]}$. 与儿童相比, T1DM成年患者的NAFLD患病率更高. 儿童与成年人 之间NAFLD患病率的差异可归因于成人更高的BMI 和更长的DM病程等因素 ${ }^{[14]}$.

在妊娠期间首次发生或发现的糖耐量减低或糖尿 病称为妊娠期糖尿病 (gestational diabetes mellitus, GDM). GDM代表妊娠期间机体的对血糖调节能力下 降, 未能适应妊娠期间的人体代谢需求. 一项队列研 究观察了GDM患者和普通人在怀孕后 25 年时NAFLD 的患病有无差异 ${ }^{[15]}$. 结果表明, 在校正了其他早期代谢 危险因素之后, GDM患病史与中年时NAFLD的发生 密切相关, 并可能通过GDM妊娠后发生的DM介导.

\section{NAFLD和DM相关的发病机制}

从流行病学研究可以看出, DM的存在对NAFLD 的发生发展起促进作用. 因其病因不同而存在差异, NAFLD的发病机制复杂, 但是二次打击学说目前已被 广泛接受. 在该学说中, 胰岛素抵抗, 过度的氧化应激 以及炎症反应在NAFLD的发生发展中起到了重要的 作用. 而二者均与DM有着密切的关系.

NAFLD的发病机制是多因素的, 其中胰岛素抵抗 是疾病病理生理学的基石. 而胰岛素抵抗和DM有密 切的关系, 是DM主要的表现之一. 脂肪酸的主要来源 来自脂肪组织内的脂肪分解, 饮食摄入和从头合成. 在 肝脏中, 游离脂肪酸(free fatty acids, FFAs)可以重新酯 化为甘油三酯, 进行 $\beta$-氧化或作为极低密度脂蛋白 (very low density lipoprotein, VLDL)排泄. 在DM的进 展中, 所有这些生理过程都会被打乱. 因为胰岛素抵抗 会使胰岛素抑制肝糖输出的能力下降，导致FFAs向肝 脏的通量增加以及甘油三酯的产生增加, 从而形成 NAFLD. 在高胰岛素血症中, SREBP-1c的上调导致糖 异生基因的过度表达, 导致脂质积聚到肝中, 从而形成 
$\mathrm{NAFLD}^{[16]}$.

脂肪酸的堆积在DM的发生发展过程中起到重要 作用. FFA过度沉积, 而脂肪酸特别容易发生氧化损伤, 形成高反应性的脂质过氧化物. 同时伴有细胞因子、 线粒体解耦联蛋白以及Fas配体的活化, 进而使脂肪变 性的肝细胞发生炎症、坏死. 持续存在的脂肪性肝炎 诱发肝细胞外基质的生成，形成脂肪性肝纤维化和 (或)脂肪性肝硬化. 在这个过程中, 炎症通路相关基因 会被激活, 表达增加. 肿瘤坏死因子- $\alpha$ (TNF- $\alpha)$, IL-1和 IL-6等脂肪因子和炎症因子在此炎症过程中起到主要 作用 ${ }^{[17]}$. 脂联素是一种具有强烈抗炎作用的脂肪因子, 对胰岛素受体具有强烈的敏化作用，已被证实在 NAFLD中受到抑制 ${ }^{[18]}$. 肝转录因子碳水化合物应答元 件结合蛋白(ChREBP)的研究也显示, 在肝脏内葡萄糖 和脂质体内平衡控制中起到了一定的作用 ${ }^{[19]}$.

\section{NAFLD和DM并发症的关系}

目前NAFLD和DM并发症之间的关系集中在 NAFLD对DM并发症发生发展的影响方面. 而对于DM 并发症出现之后, 是否促进了NAFLD的发生以及进展 则没有研究.

在大血管并发症方面, 2017年, 中国一个大型队列 研究中发现，在校正了常规CVD危险因素、DM病 程、 $\mathrm{HbA1c}$ 水平、胰岛素抵抗、血清肝酶水平和药物 的使用情况后, 超声诊断的NAFLD与颈动脉和下肢动 脉弹样硬化斑块的患病率增加有关 ${ }^{[20]}$. 无独有偶, 在意 大利的Valpolicella DM心脏研究中, 纳入意大利门诊 2392例无慢性肝病继发原因的DM患者. 在这些DM患 者中, 使用超声诊断确诊为NAFLD的患者, 冠状动 脉、脑血管和外周血管疾病的发生率明显高于无 NAFLD的患者. 当校正年龄、性别、BMI、腰围、吸 烟状况、LDL水平、 $\mathrm{HbA} 1 \mathrm{c}$ 水平、 $\mathrm{DM}$ 病程，是否有 MetS以及降血糖药、降压药、降脂药和抗血小板药 使用情况后, 以上疾病的患病率仍有统计学差异 ${ }^{[14]}$. 此 外,一些研究也表明, NAFLD与DM慢性血管并发症风 险增加密切相关，同时患有DM和NAFLD的患者其他 并发症发生的几率会增加. 在一项纳入了 34000 人的 中位随访时间为 6.9 年的meta分析中, NAFLD与致死 性、非致死性CVD事件风险增加近 $65 \%$ 有关。以上的 研究为NAFLD与DM大血管并发症之间的关系提供了
强有力的证据.

除了大血管并发症之外，NAFLD还增加了DM患 者微血管并发症的风险. Valpolicella DM心脏队列研 究中 ${ }^{[21]}$, 纳入 2103 名T2DM患者, 基线时排除已知有慢 性肝病或心血管疾病的 T2DM患者. 诊断为NAFLD患 者出现DM肾病或者视网膜病变(增殖性或激光治疗) 的风险是非NAFD患者的接近两倍，该分析校正了年 龄、性别、BMI、腰围、吸烟状况、高血压、DM病 程、 $\mathrm{HbA1}$ 水平、血浆血脂水平和药物使用情况. 其 他研究表明, 经超声或组织学诊断的NAFLD, NAFLD 的存在及其严重程度与 T2DM或DM前期患者中异常 蛋白尿的患病率增加或肾功能下降密切相关 ${ }^{[22]}$. 为进 一步了解DM患者中NAFLD和微血管并发症的风险, 需要进行长时间的前瞻性研究来阐明其二者的关系. Valpolicella DM心脏队列研究的一个亚组也进行了 T2DM患者中NAFLD患病与否与慢性肾病 (chronic kidney disease, CKD)的风险之间的研究 ${ }^{[23]}$. 该研究随 访了1760名肾功能正常或接近正常且无明显蛋白尿的 门诊T2DM患者. 随访期间, 547名参与者发生CKD. 研 究表明, NAFLD与CKD风险适度增加有关(OR： 1.69 , $95 \%$ CI: 1.3 2.6; $P<0.001)$. 当校正性别、年龄、体重 指数、腰围、血压、吸烟、DM病程、糖化血红蛋 白、血脂、基线 GFR、微量白蛋白尿和药物 (降糖 药、降脂药、抗高血压药或抗血小板药)后, 没有明显 减弱相关性(OR：1.49；95\% CI: 1.1 2.2；P<0.01). Targher等人也进行了T1DM患者中，NAFLD和微血管 病变相关性的研究. 经过平均时间为 5.2 年的随访后, 261中的61名患者进展为CKD. 结果显示, NAFLD与 CKD发生风险增加相关(OR: $2.85,95 \%$ CI: 1.59 5.10; $P<0.001)$. 当校正其他相关危险因素后, 没有明显减弱 该相关性(OR: $2.03,95 \% \mathrm{CI}: 1.10 \sim 3.77, P<0.01)$. 当将 NAFLD添加到CKD的危险因素后，显着提高了预测 $\mathrm{CKD}$ 的回归模型的预测能力 $(\mathrm{OR}$ ： $0.79,95 \% \mathrm{CI}$ : 0.73 0.86 (加入NAFLD) vs. OR： 0.76, 95\% CI: 0.71 0.84 (未加入NAFLD), $P=0.002$ ). 该研究证明, NAFLD与CKD发病率增加密切相关的研究. NAFLD 为T2DM患者独立于传统的心肾危险因素的危险因素. 总之, 多个研究结果表明, 非酒精性脂肪性肝病与 $\mathrm{T} 2 \mathrm{DM}$ 患者CKD发病率增加有关，与许多基线混杂因 素无关.

关于NAFLD和糖尿病周围神经病变的研究较少. 
最近在一项纳入了 286 名 T1DM的患者的队列研究中, 患有NAFLD的患者的糖尿病神经病变的发生率是无 NAFLD的T1DM患者的接近6倍 ${ }^{[24]}$. 而这项观察的结果 在校正了年龄、性别、吸烟状况、糖尿病持续时间、 $\mathrm{HbA} 1 \mathrm{c}$ 水平、 $\mathrm{CKD}$ 以及既往缺血性心脏病后仍有 意义.

以上研究表明，NAFLD增加了DM患者并发症风 险. 除了NAFLD本身会造成胰岛素抵抗，使血糖控制 变差从而增加并发症的风险之外，还需要考虑和 NAFLD密切相关的其他器官引起的并发症发生风险 增加的可能. 例如, 肝脏与脂肪组织之间的密切关系, NAFLD可以导致慢性炎症的发生发展，以及在凝血和 纤溶系统调节中的作用. 而机体慢性炎症以及凝血、 纤溶系统的调节失常是大血管以及微血管并发症的重 要机制之一.

\section{4 降糖药与NAFLD}

DM和NAFLD预防与干预方面，运动和控制饮食 均起到了重要的作用. 目前在NAFLD方面，除了运动 和饮食控制外，没有专门针对NAFLD的药物. 虽然降 低体重是NAFLD的重要治疗手段之一, 但是目前用于 减肥的药物还没有用于NAFLD的治疗. DM患者中 NAFLD的患病率高，且胰岛素抵抗在NAFLD的发生 发展中起到了重要的作用. 由于没有针对NAFLD的药 物治疗. 只有几类降糖药针对是否能缓解DM患者中 NAFLD的程度做了研究.

在常用的降糖药物中, 双胍类和噻唑烷二酮类药 物可以有效改善胰岛素抵抗. 所以这两类药物较早被 用来作为 NAFLD的药物治疗. 虽然在DM的治疗中, 二甲双胍以减重和改善胰岛素抵抗作为主要特点, 但 是在一项 meta分析中(其中 $27 \%$ 为DM的大型荟萃分析 中), 尽管 $\mathrm{HbA1} 1 \mathrm{c}$ 和体重有所改善, 但是在肝脏脂肪变 性或炎症方面没有显著统计学的组织学改善. 另外一 项将二甲双胍和吡格列酮进行对比治疗NAFLD的研 究显示, 经过 4 个月治疗后，吡格列酮和二甲双胍均显 著降低了肝功能实验结果的水平，以及血脂、肝脏含 量等指标 ${ }^{[25]}$. 在PIVENS研究的 247 名被诊断为NASH 的非DM患者在使用吡格列酮(维生素E及安慰剂作为 对照)进行治疗了96周后，包括NAFLD活动评分以及 气球样评分的主要结果均出现改善, 而纤维化没有恶
化 ${ }^{[26]}$. Belfort等人 ${ }^{[27]}$ 也对DM前期或T2DM患者使用吡 格列酮治疗后，观察到肝脂肪变性和坏死性炎症显着 改善, 脂肪肝可在 6 个月内逆转. 二甲双胍和吡格列酮 对于NAFLD的缓解可能和其降低外周以及肝脏组织 的胰岛素抵抗的作用有关. 最新研究表明, 在肥胖小鼠 (Mus musculus) 中二甲双胍可能通过apoA5和AMPK/ LXR $\alpha$ 信号通路缓解肥胖相关脂肪肝 ${ }^{[28]}$. 另外一项研 究则指出, 二甲双胍可能通过脂肪肝相关的IncRNA以 及 circRNA等途径改善NAFLD ${ }^{[29]}$. 而对于吡格列酮对 NAFLD的缓解作用可能的机制并没有基础方面的 研究.

另外, 较新的降糖药DPP-4抑制剂以及GLP-1类似 物也进行了NAFLD治疗相关的研究. 一项持续 24 周的 西格列汀的随机对照研究表明, NAFLD的患者使用西 格列汀是安全的，每天服用 $100 \mathrm{mg}$ 西格列汀不能更好 地改善NAFLD患者的肝脏纤维化评分 ${ }^{[30]}$. 另外一项研 究也显示, 西格列汀虽然很好地降低了血糖, 但是没有 比对照组更好的降低转氨酶 ${ }^{[31]}$. GLP-1类似物对于DM 以及减重都有很好的疗效. 目前一项临床研究显示, 与 改善生活方式组相似, 利拉鲁肽组的体重降低, 同时转 氨酶水平也得到改善 ${ }^{[32]}$. 目前, 在随机对照研究中唯一 经过FDA批准的可显著改善DM患者肝脏组织学改变 的药物有吡格列酮和利拉鲁肽. 关于利拉鲁肽进行 NAFLD治疗的研究还在进行中. 有基础研究表明, 利 拉鲁肽可能通过抑制氧化应激和炎症反应来缓解 NAFLD的进展 ${ }^{[33]}$. 另外利拉鲁肽也可能通过改善线粒 体的结构, 通过SIRT1/SIRT3-FOXO3a信号通路来改善 NAFLD ${ }^{[34]}$. GLP-1类似物对NAFLD的改善在临床和基 础研究中均得到较好的验证. 鉴于其在NAFLD患者使 用中的安全性以及有效性, GLP-1类似物有望可以成 为NAFLD的治疗药物. 当然在这之前需要进行更多的 临床研究尤其是随机对照试验来验证其有效性以及安 全性。

\section{5 总结}

DM和NAFLD已经成为全球高发的疾病，给社会 以及医疗造成巨大的负担. DM在NAFLD的发生发展 中起到推动作用，而在DM患者中NAFLD为其并发症 发生的独立危险因素. DM患者中可能出现肝酶变化 不明显的NAFLD，且DM患者中NASH的诊断比例较 
高. DM患者中NAFLD的篎查应得到内分泌科医师的

重视．医务人员进行普及其重要性以及早实施生活方 式和药物干预措施是必不可少的. 到目前为止，生活 方式干预，减重仍然是NAFLD和DM协同管理的黄金
标准. 虽然药物治疗起到重要作用，但对于健康人群 进行普及预防，改善一般人群的生活方式，从非患病 人群开始杜绝营养过剩以及缺乏运动仍不失为重要的 手段.

\section{参考文献}

1 International Diabetes Federation. IDF Diabetes Atlas, 8th ed. (http://www.diabetesatlas.org)

2 Stumvoll M, Goldstein B J, van Haeften T W. Type 2 diabetes: principles of pathogenesis and therapy. Lancet, 2005, 365: 1333-1346

3 Wild S, Roglic G, Green A, et al. Global Prevalence of Diabetes: estimates for the year 2000 and projections for 2030. Diabetes Care, 2004, 27: $1047-1053$

4 Guariguata L, Whiting D R, Hambleton I, et al. Global estimates of diabetes prevalence for 2013 and projections for 2035. Diabetes Res Clin Pract, 2014, 103: 137-149

5 Portillo-Sanchez P, Bril F, Maximos M, et al. High prevalence of nonalcoholic fatty liver disease in patients with type 2 Diabetes mellitus and normal plasma aminotransferase levels. J Clin Endocrinol Metab, 2015, 100: 2231-2238

6 Leite N C, Salles G F, Araujo A L E, et al. Prevalence and associated factors of non-alcoholic fatty liver disease in patients with type-2 diabetes mellitus. Liver Int, 2009, 29: 113-119

7 Leite N C, Villela-Nogueira C A, Pannain V L N, et al. Histopathological stages of nonalcoholic fatty liver disease in type 2 diabetes: prevalences and correlated factors. Liver Int, 2011, 31: 700-706

8 Fracanzani A L, Valenti L, Bugianesi E, et al. Risk of severe liver disease in nonalcoholic fatty liver disease with normal aminotransferase levels: a role for insulin resistance and diabetes. Hepatology, 2008, 48: 792-798

9 Neuschwander-Tetri B A, Clark J M, Bass N M, et al. Clinical, laboratory and histological associations in adults with nonalcoholic fatty liver disease. Hepatology, 2010, 52: 913-924

10 Nascimbeni F, Pais R, Bellentani S, et al. From NAFLD in clinical practice to answers from guidelines. J Hepatology, 2013, 59: 859-871

11 El-serag H B, Tran T, Everhart J E. Diabetes increases the risk of chronic liver disease and hepatocellular carcinoma. Gastroenterology, 2004, 126: $460-468$

12 Serra-Planas E, Aguilera E, Castro L, et al. Low prevalence of non-alcoholic fatty liver disease in patients with type 1 diabetes is associated with decreased subclinical cardiovascular disease. J Diabetes, 2017, 9: 1065-1072

13 El-Karaksy H M, Anwar G, Esmat G, et al. Prevalence of hepatic abnormalities in a cohort of Egyptian children with type 1 diabetes mellitus. Pediatric Diabetes, 2010, 11: 462-470

14 Targher G, Bertolini L, Padovani R, et al. Prevalence of nonalcoholic fatty liver disease and its association with cardiovascular disease among type 2 diabetic patients. Diabetes Care, 2007, 30: 1212-1218

15 Ajmera V H, Gunderson E P, VanWagner L B, et al. Gestational diabetes mellitus is strongly associated with non-alcoholic fatty liver disease. Am J Gastroenterol, 2016, 111: 658-664

16 Samuel V T, Petersen K F, Shulman G I. Lipid-induced insulin resistance: unravelling the mechanism. Lancet, 2010, 375: 2267-2277

17 Jou J, Choi S S, Diehl A M. Mechanisms of disease progression in nonalcoholic fatty liver disease. Semin Liver Dis, 2008, 28: 370-379

18 Whitehead J P, Richards A A, Hickman I J, et al. Adiponectin — a key adipokine in the metabolic syndrome. Diabetes Obes Metab, 2006, 8: 264280

19 Uyeda K, Repa J J. Carbohydrate response element binding protein, ChREBP, a transcription factor coupling hepatic glucose utilization and lipid synthesis. Cell Metab, 2006, 4: 107-110

20 Guo K, Zhang L, Lu J, et al. Non-alcoholic fatty liver disease is associated with late but not early atherosclerotic lesions in Chinese inpatients with type 2 diabetes. J Diabetes Compl, 2017, 31: 80-85

21 Targher G, Bertolini L, Rodella S, et al. Non-alcoholic fatty liver disease is independently associated with an increased prevalence of chronic kidney disease and proliferative/laser-treated retinopathy in type 2 diabetic patients. Diabetologia, 2008, 51: 444-450

$22 \mathrm{Li} \mathrm{Y,} \mathrm{Zhu} \mathrm{S,} \mathrm{Li} \mathrm{B,} \mathrm{et} \mathrm{al.} \mathrm{Association} \mathrm{between} \mathrm{non-alcoholic} \mathrm{fatty} \mathrm{liver} \mathrm{disease} \mathrm{and} \mathrm{chronic} \mathrm{kidney} \mathrm{disease} \mathrm{in} \mathrm{population} \mathrm{with} \mathrm{prediabetes} \mathrm{or}$ 
diabetes. Int Urol Nephrol, 2014, 46: 1785-1791

23 Targher G, Chonchol M, Bertolini L, et al. Increased risk of CKD among type 2 diabetics with nonalcoholic fatty liver disease. J Am Soc Nephrol, 2008, 19: 1564-1570

24 Mantovani A, Rigolon R, Mingolla L, et al. Nonalcoholic fatty liver disease is associated with an increased prevalence of distal symmetric polyneuropathy in adult patients with type 1 diabetes. J Diabetes Compl, 2017, 31: 1021-1026

25 Razavizade M, Jamali R, Arj A, et al. The effect of pioglitazone and metformin on liver function tests, insulin resistance, and liver fat content in nonalcoholic fatty liver disease: a randomized double blinded clinical trial. Hepat Mon, 2013, 13: e9270

26 Bell L N, Wang J, Muralidharan S, et al. Relationship between adipose tissue insulin resistance and liver histology in nonalcoholic steatohepatitis: a pioglitazone versus vitamin E versus placebo for the treatment of nondiabetic patients with nonalcoholic steatohepatitis trial follow-up study. Hepatology, 2012, 56: 1311-1318

27 Belfort R, Harrison S A, Brown K, et al. A placebo-controlled trial of pioglitazone in subjects with nonalcoholic steatohepatitis. N Engl J Med, 2006, 355: 2297-2307

28 Lin M J, Dai W, Scott M J, et al. Metformin improves nonalcoholic fatty liver disease in obese mice via down-regulation of apolipoprotein A5 as part of the AMPK/LXRalpha signaling pathway. Oncotarget, 2017, 8: 108802-108809

29 Guo J, Zhou Y, Cheng Y, et al. Metformin-induced changes of the coding transcriptome and non-coding RNAs in the livers of non-alcoholic fatty liver disease mice. Cell Physiol Biochem, 2018, 45: 1487-1505

30 Joy T R, McKenzie C A, Tirona R G, et al. Sitagliptin in patients with non-alcoholic steatohepatitis: a randomized, placebo-controlled trial. World J Gastroenterol, 2017, 23: 141-150

31 Deng X L, Ma R, Zhu H X, et al. Short article. Eur J Gastroenterol Hepatol, 2017, 29: 297-301

32 Khoo J, Hsiang J, Taneja R, et al. Comparative effects of liraglutide $3 \mathrm{mg}$ vs structured lifestyle modification on body weight, liver fat and liver function in obese patients with non-alcoholic fatty liver disease: a pilot randomized trial. Diabetes Obes Metab, 2017, 19: 1814-1817

33 Gao H, Zeng Z, Zhang H, et al. The glucagon-like peptide-1 analogue liraglutide inhibits oxidative stress and inflammatory response in the liver of rats with diet-induced non-alcoholic fatty liver disease. Biol Pharmaceut Bull, 2015, 38: 694-702

34 Tong W, Ju L, Qiu M, et al. Liraglutide ameliorates non-alcoholic fatty liver disease by enhancing mitochondrial architecture and promoting autophagy through the SIRT1/SIRT3-FOXO3a pathway. Hepatol Res, 2016, 46: 933-943

\title{
Diabetes mellitus and non-alcoholic fatty liver disease
}

\author{
WANG Qian \& ZHAO JiaJun \\ Department of Endocrinology, Shandong Provincial Hospital affiliated to Shandong University, Jinan 250021, China
}

The prevalence of non-alcoholic fatty liver disease (NAFLD) is high in patients with diabetes mellitus (DM). Diabetes mellitus, especially type 2 diabetes mellitus (T2DM), speeds up the progression of NAFLD. And patients with DM have no significant increase in liver enzymes whether diagnosed NAFLD or not. NAFLD is an independent risk factor for the development of macrovascular and microvascular complications in diabetic patients. Thiazolidinediones, DPP-4 inhibitors, and GLP-1 analogs can effectively relieve the degree of fatty liver in diabetic patients. When they have received long-term treatment, their fatty livers could be resolved.

\section{diabetes, non-alcoholic fatty liver disease, diabetes complications, antidiabetic drugs}

doi: $10.1360 / \mathrm{N} 052018-00042$ 